\title{
Promoting sustainable proliferation of NK cells by up-regulating IL2 and IL12 through CRISPR-CAS9Promoting sustainable proliferation of NK cells by up- regulating IL2 and IL12 through CRISPR-CAS9
}

\author{
Changxin Huang \\ The Affiliated hospital of Hangzhou Normal University \\ Lili Yang \\ The Affiliated hospital of Hangzhou Normal University \\ Congjie Wang \\ Lin'an District People's Hospital; \\ Lanlan Gao \\ Hangzhou Normal University School of Medicine \\ Ying Zhu \\ Zhejiang Traditional Chinese Medicine Hospital \\ Yongqiang $\mathrm{Li}$ \\ The Affiliated hospital of Hangzhou Normal University \\ Yanfei Shi \\ Hangzhou Normal University School of Medicine \\ Siyu Zhang \\ The Affiliated hospital of Hangzhou Normal University \\ Zuanmin Ge \\ Hangzhou Normal University School of Medicine \\ Meng Su \\ Hangzhou Normal University School of Medicine \\ Xuechun Wang \\ Hangzhou Normal University School of Medicine \\ lingzhi chen ( $\square$ chenlingzhitongxu@126.com ) \\ Jiaxing First Hospital https://orcid.org/0000-0001-5983-9706
}

\section{Research Article}

Keywords: NK92 cells, crispr/cas9 gene editing, IL2, IL12, Sustainable proliferation

Posted Date: March 30th, 2021

DOI: https://doi.org/10.21203/rs.3.rs-317025/v1

License: (c) (1) This work is licensed under a Creative Commons Attribution 4.0 International License. Read Full License 


\section{Abstract}

NK cells are an important part of human immunity and are often used in clinical antitumor adoptive cell immunotherapy. However, the small number and low activity of NK cells in the tumor microenvironment limit its clinical efficacy.Studies have found that cytokines IL2 and IL12 play key roles in the proliferation of NK cells and the improvement of the activity of killing tumor cells, the toxic and side effects, however, are relatively large when the systemic application reaches the effective concentration. To study the effect of autocrine secretion of IL2 and IL12 on proliferation and anti-tumor activity of NK92 cell lines by editing NK cells' genes, and to construct NK92 cells with sustainable proliferation. Lentivirus was transfected into NK92 cells through the synergistic effect of crispr/cas9 localization gene editing technology and VP64 transcriptional activation domain to construct NK92-IL2-IL12 cell lines. The expression levels of IL2 and IL12 in the cell lines were detected by qPCR and ELISA.NK92 cell lines were inoculated into mouse tumor and the proliferation and anti-tumor effect of NK cells in tumor were observed. The vector plasmids IL2 and IL12 were successfully transferred into NK92 cells, which activated the up-regulated expression of IL2/IL12 in NK92, significantly increased the activity and lethality of NK92 cells, and sustained the proliferation of NK92 cells in mouse tumor.IL2 and IL12 can significantly promote the proliferation and killing activity of NK92 cell lines through autocrine, which have stronger anti-tumor activity than the wild-type ones, with continuous proliferation in the tumor.

\section{Introduction}

NK cells are an important part of human immunity and have strong innate immunity. Studies have found that the number of NK cells that reflect the immune function of the body is closely related to the prognosis of patients, but with the progression of tumor, the number of NK cells in patients often decreases significantly [1]. NK cells can recognize and kill malignant cells without pre-sensitization [2, 3]; adoptive transferred NK cells seem to have the lowest targeted toxicity spectrum [4], avoiding dangerous cytokine recruitment, which usually occurs in many T cell-based immunotherapy [4]. No obvious side effects were found in the clinical application of allogeneic NK cells in non-patients. Therefore, it is often used in clinical anti-tumor adoptive cellular immunotherapy, and has become an effective means to enhance the traditional treatment of cancer[5]. For example, it has been successfully used in the treatment of hematological diseases such as leukemia [6-8]. And the treatment of solid tumors such as melanoma and[2]metastatic neuroblastoma [9, 10]. However, the therapeutic effect on other solid tumors such as colorectal cancer is less impressive [3]. The possible reason is that the small number and low activity of NK cells in the tumor microenvironment restrict its clinical curative effect, and the related research focuses on promoting its in vivo survival, killing activity and the efficiency of migration to the tumor site $[3,4,1,6]$. These strategies are important advances in the emerging field of clinical NK cellular immunotherapy $[4$, 11]. Among them, the most important thing is the sustained steady-state proliferation and enhanced activity in the tumor, which is rarely reported at present. Therefore, the sustainable expansion and enhanced activity of NK cells in tumor are highly attractive as a means to inhibit or eliminate the growth of unconstrained tumor cells[12]. A great deal of evidence shows that how to improve the killing activity of NK cells in tumor microenvironment and the stable and sustainable proliferation of NK cells in tumor is the key link that restricts the curative effect.

IL12 is a cytokine with direct inhibitory effect on tumor, which is composed of two subunits of p35 and p40. At the same time, it can make NK cells proliferate continuously and be in a state of cellular immune response that can effectively play an anti-tumor effect [13]. The proliferation of NK cells also depends on IL2 stimulators. Studies have found that cytokines IL2 and IL12 play a key role in the proliferation of NK cells and improve the activity of killing tumor cells, but the toxicity and side effects become more serious and intolerable when these cytokines are used in the whole body at an effective concentration. Therefore, NK cells are usually isolated and cultured in vitro and then infused in vivo to treat tumors. However, recent studies have shown that NK cells activated by cytokines and expanded in vitro lack or inadequately express homing receptors; such adoptive natural NK cells rarely enter and expand in tumors.

The CRISPR/Cas9 system is a genome editing technology that has been widely used in humans [20-21], mice [22], zebrafish [23-24] and other different species using RNA-guided nuclease to modify genomic DNA. , can achieve targeted and precise genetic modification in any animal. It is widely used in gene knockout, gene insertion, gene site-directed mutation, gene function inhibition, animal breed breeding and human disease animal model creation, etc., providing a new direction for gene therapy.In 2012, Jinek et al [12] transformed the CRISPR/Cas9 system, which contains two non-coding RNA crRNA and tracrRNA, into a single-directed RNA (Single-guide RNA, sgRNA), which can guide Cas9 proteins to carry out targeted double-strand cleavage of specific DNA sequences (directly to the upstream 3 bp of DNA double-strand containing PAM (Protospacer adjacent motif) NGG); to form a (Double strand breaks,DSBs with a blunt end DNA double-strand break at a specific location). It was found that the localization function of the CRISPR/Cas9 system combined with the fusion VP16 tetramer activator VP64 could directly up-regulate the transcriptional level of endogenous genes [14]. In this study, through the localization function of CRISPR/Cas9 in the genome, the dCas9-VP64 fusion protein was constructed, and the expression of the target gene was up-regulated under the guidance of sgRNA localization $[15,16]$. In this study, we used this gene editing technique to make NK92 cell line constantly up-regulate the overexpression of cytokines IL2 and IL12, on NK92 cells by autocrine or paracrine, which could not only improve the cytotoxic activity of NK92 cells, but also promote its continuous proliferation and avoid the side effects caused by systemic infusion of cytokines.

\section{Materials And Methods}

\subsection{Materials}

\subsubsection{Cell lines and plasmids}

The NK-92 cell lines used in this study were donated by Professor Park Zhenghao of Hangzhou normal University (purchased from ATCC); 293 T and K562 cell lines and preserved in the laboratory; Ecoli-DH5 a competent cells were purchased by Shanghai Biological Engineering Co., Ltd.; lentivirus packaging plasmids ps PAX2 and PMD2.G laboratory provided. The core plasmids dcas9-vp64 (LW415) and grnalL2/IL12 (LW593) were synthesized by Beijing Hesheng Genetics Company.

\subsection{2 main materials}

a-MEM medium (gibco); fetal bovine serum (gibco Australia); horse serum (gibco); IL2 (peprotech); penicillin streptomycin (Beijing, China); SCID mice (T and B lymphocyte deficient animals), 4murine 6 weeks old, (purchased from Jiangsu Cuiji Extractive Pharmaceutical Kang Biology Co., Ltd., Nanjing, China); IL12 
and IL2 Elisa kits (gibco biology, Hangzhou); qPCR kit (Takara); Rabbit anti-human CD56 antibody (ab75813,Abcam) and goat anti-rabbit IgG (ab150077, Abcam); qPCR primers were synthesized by Shanghai Biological Engineering Co., Ltd.; CCK8, trypsin, PBS buffer, PEG8000 and other related equipment were purchased from Bao Riyi Biotechnology Engineering (Beijing) Co., Ltd. And Boshi Biological Co., Ltd. The red fluorescent protein labeling kit was purchased from Shanghai Zhongqiao Xinzhou Biological Co., Ltd.

\section{2 method}

\subsubsection{Cell culture}

293 T cells: $10 \%$ fetal bovine serum, $1 \%$ penicillin DMEM medium, $6 \mathrm{~cm}, 10 \mathrm{~cm}$ petri dish culture; K562 cells: cultured in RPMI 1640 medium containing $10 \%$ fetal bovine serum and 1\% penicillin streptomycin in T25 culture bottle; NK92 cells: $12.5 \%$ fetal bovine serum, $12.5 \%$ horse serum, $1 \%$ penicillin streptomycin, a-MEM medium of $100 \mathrm{U} / \mathrm{ml} \mathrm{IL2}$, T2 25 culture bottle; The culture medium was cultured in $37^{\circ} \mathrm{C}$ and $5 \% \mathrm{CO} 2$ incubator, and the culture medium was changed for 2 days.

\subsubsection{Design of gRNA primers}

The 5KB DNA fragments upstream of transcriptional initiation site of IL-2 target gene (GenBank number: NM_000586), IL-12A target gene (GenBank number: NM_000882) and IL-12B target gene (GenBank number: NM_002187) were obtained from the database of (NCBI) of the National Information Technology Center of the United States. SgRNA, selected the activated gRNA sequence with good efficacy and specificity from the website (http://crisprera.Stanford.edu/). The miss effect is predicted through the website, and the sequences of gRNA:IL2 gRNA, IL12A gRNA and IL12B gRNA with the lowest miss effect are shown in Table 1, respectively. The AA base only plays a terminating role through two $A$ base intervals, and its gene sequence is: GGATCTGGCGCCACCAACTTCTCTCTGCTGAAGCAGGCCGGCGACGTGGAGGAGAACCCAGGCCCA; Beijing Hesheng Gene Company was entrusted to synthesize IL2-IL12A-IL12B gRNA;PCR and amplify double-stranded DNA, to form double-stranded DNA,. The PUC57vetcor, was digested by Pacl,Agel restriction enzyme and then combined with linear skeleton PUC57vetcor by T4 DNA ligase to construct lentiviral vector (pLV-U6-gRNA1- U6-gRNA2-U6-gRNA3CMV-EGFP). Among them, pLV is lentiviral plasmid, U6 is U6 promoter sequence, gRNA1, gRNA2, gRNA3 represent IL2 gRNA, IL12A gRNA, IL12B gRNA,CMV represent $\mathrm{CMV}$ promoter sequence, EGFP represents green fluorescent protein sequence. The recombinant vector was used to transform $\mathrm{DH} 5 \mathrm{a}$ competent cells, and the clones were selected, and the plasmids were extracted and sequenced. High purity extracted plasmid, SSA report vector (skeleton PUC57 plasmid) was purchased from Beijing Hesheng Gene Co., Ltd.; item number: A-FW-201807200313.

\begin{tabular}{|ll|}
\hline IL2-gRNA-PF & GGATCTCCTCAAGTGTCCCC \\
\hline IL2-gRNA-PR & GGGGACACTTGAGGAGATCC \\
\hline IL12A-gRNA-PF & GCCGACGTTGCACCAGGTGC \\
\hline IL2A-gRNA-PR & GCACCTGGTGCAACGTCGGC \\
\hline IL2B-gRNA-PF & TCCTCGTTATTGATACACAC \\
IL2B-gRNA-PR & GTGTGTATCAATAACGAGGA \\
\hline
\end{tabular}

Table1 sgRNA(single guide RNA囚primer design

\subsubsection{Construction of NK92-IL2-IL12 cell line}

1.2.2.1 Construction of lentiviral vector plasmids of IL2 and IL12.

1) Design gRNA primers (Table 1). On the basis of previous experiments, refer to the website http://crispr-era.Stanford. Edu/, selected gRNA with better specificity and efficacy.

2) Entrusting Syngenics Company to synthesize viral vector plasmids dcas9-vp64 (LW415) 『and gRNA IL2 / IL12 (LW593)according to gRNA synthesis. The generation of lentiviral vectors is schematically presented in Fig 1a-b.

3) The two plasmids were synthesized and transferred to DH5- a competent cells, then centrifuged at $37^{\circ} \mathrm{C}$ for 3 hours, $100 \mathrm{ul}$ coated plate was taken, incubator was incubated overnight at $37^{\circ} \mathrm{C}$, larger strains were selected, and the larger strains were amplified by $150 \mathrm{Met} 200 \mathrm{rpm} / \mathrm{min}$ at $37^{\circ} \mathrm{C}$.

4) Plasmid extraction kit, DNA concentration was measured and stored at- $20^{\circ}$.

5) Identification of positive clones: three or four single bacteria were selected and the plasmid DNA was extracted and sequenced. The primer for sequencing was CAGGAAGAGGGCCTATTTCCC.

6) The identified plasmid is used as the target plasmid for subsequent transfection.

\subsubsection{2 lentivirus plasmid packaging and virus concentration.}

293 T cells were cultured in 6-well plate (DMEM medium, 10\% FBSpl 1\% penicillin streptomycin), the cell density reached $70 \% 80 \%$, and the adhesion was uniform, and the growth state was good; Lipofectamine 3000 transfection reagent, according to A solution: lipo3000 $3.75 \mathrm{ul}$, a-MEM medium (serum-free) 121.25ul liquid B: a-MEM medium, psPAX2 1.5ug, PMD2.G 0.5ug, LW415 2ug, LW593 2ug P3000 12ul (2ul/ug) ratio configuration mixture; After 5 minutes, liquid $B$ was added to liquid $A$, and after 20 minutes, 293T cell culture medium was added evenly, and the fluorescence effect was observed under fluorescence microscope after 48 hours and 96 hours. $293 \mathrm{~T}$ cells and their culture medium were collected and centrifuged at $4{ }^{\circ} \mathrm{C} 3000 \mathrm{~g}$ for 20 minutes. The supernatant containing lentivirus was taken.

The virus supernatant was collected and filtered by $0.45 \mathrm{um}$, and $5 x$ PEG8000 mother liquid after $7.5 \mathrm{ml}$ sterilization was added to each $30 \mathrm{ml}$, and the virus solution was mixed with each $30 \mathrm{~min}$ for 5 times, standing overnight at $4{ }^{\circ} \mathrm{C}$, centrifuging for 20 minutes at $4{ }^{\circ} \mathrm{C}$, and dissolving the virus solution with a certain amount of PBS. The virus suspension was packed into $50 \mathrm{ul}$ each and stored at- $80{ }^{\circ} \mathrm{C}$.

\subsubsection{3 determination of virus titer.}

The day before detection, 293T cells were passaged, $1 \times 104$ cells were added to each well in 96-well plate and cultured at $37^{\circ} \mathrm{C}$ for 24 hours. Before 
transduction, the cells digested in one hole were counted and the cell quantity was obtained. the supernatant of 100ul virus was mixed in the medium of $100 \mathrm{ul}$ (DMEM+10\%FBS). After mixing, it was labeled as 10murl, and then diluted with 10 times gradient until diluted to 10 mur8. (6 wells in each group), take $1 \mathrm{ul}$ virus concentrate and 200ul medium (DMEM+10\%FBS), after mixing, mark it as 10 Mel 1, then dilute it with 10 times gradient until diluted to 10 mi 8 . (6 holes in each group), select the desired cell pores, absorb the culture medium from the culture pores, add the gradient diluted virus solution 190 ul, and gently mix it, and then culture it in the $5 \% \mathrm{CO} 2$ incubator at $37^{\circ} \mathrm{C}$. After 24 hours, the number of fluorescent cells in each well was observed under fluorescence microscope and counted (the culture medium was absorbed and washed twice by PBS before taking pictures). The fresh complete medium $200 \mathrm{ul}$ was changed and cultured for 24 hours. The number of fluorescent cells in each well was observed under fluorescence microscope and counted. Virus titer (IU/mL) $=(\mathrm{number}$ of transfected cells $\times$ dilution times $\times$ percentage of infection) / (virus volume $(\mathrm{mL})$ per $\mathrm{mL}$ ). Virus concentrate titer $(\mathrm{IU} / \mathrm{mL})=1.8 \times 104 \times 100 \times 3 \% \mathrm{IU} / \mathrm{mL}$,

$0.005 \times 1.08 \times 107$ virus supernatant titer $(\mathrm{IU} / \mathrm{mL})=1.8 \times 104 \times 100 \times 3 \% \mathrm{IU} / \mathrm{mL}, 0.5 \mathrm{~cm} 5.4 \times 106 \mathrm{IU} / \mathrm{mL}$.

\subsubsection{4 lentivirus transfection into NK92 cells.}

NK92 cells were cultured in T25 culture flask (a-MEM medium, 12.5\% FBS 12.5\% horse serum, 1\% penicillin streptomycin, 100U/ml IL2). The NK92 cells with good growth condition were infected, and the cell concentration was about $65 \% 80 \%$. After centrifugation, the recombinant virus solution $3 \mathrm{ml}$ (virus titer about $10^{\wedge} 6$ ) and virus concentrated solution $50 \mu \mathrm{I}$ (virus titer about $10^{\wedge} 7$ ) were taken and changed to complete medium $5 \mathrm{ml}$. The cell infection efficiency was observed under fluorescence microscope after $48 \mathrm{~h}$ and $72 \mathrm{~h}$. In order to further improve the transfection efficiency, $1 \mathrm{RV} 1000$ was added to $5 \mathrm{ul}$ polybrene to improve the infection efficiency, and the infection could be repeated at intervals of two days. After repeated screening with $5 \mathrm{ul}$ puromycin (concentration of $2 \mathrm{mg} / \mathrm{ml}$ ) added to the culture medium, the cells without infection were killed and the positive cells proliferated stably for 24 hours each time. The results showed that the green fluorescence expression level of $293 \mathrm{~T}$ cells was higher after lentivirus transfection, and the transfection rate was more than $99 \%$. After 48 hours of infection, the green fluorescence expression level of NK92 cells was found, and the infection rate was about $20 \%$. After repeated infection and repeated screening with puromycin, the positive rate was about $80-90 \%$ (Figure $1 \mathrm{C}$ ).

\subsubsection{NK92-IL2-IL12 cell line identification.}

NK92 cells in logarithmic phase lentivirus transfection group and wild type group (a-MEM culture medium, $12.5 \%$ FBScr $12.5 \%$ horse serum, $1 \%$ penicillin streptomycin, $100 \mathrm{U} / \mathrm{ml} \mathrm{IL2}(1000 \mathrm{pg} / \mathrm{ml})$ ), count $10^{\wedge} 7$, added to T2 5 culture bottle, fixed volume to $5 \mathrm{ml}$, were placed at $37^{\circ} \mathrm{C}$ and $5 \% \mathrm{CO} 2$ incubator for 48 hours, then centrifuged to take culture medium, IL2 and IL12 protein expression was detected according to ELISA kit protocol, 3 pairs of holes were set in each group. They were divided into blank medium group, factor modification group and wild type NK92 group. The cells of factor modified group and wild type NK92 group were extracted from RNA,Takara kit by Trizol method and reverse transcribed into CDNA,qPCR for quantitative detection of IL2 and IL12 gene expression. Factor modified group and wild type NK92 cell group were set up. Three groups of parallel holes were set in each group and repeated three times. QPCR results were used to observe the size of amplified fragments by gel electrophoresis and compare the size of genes.

\begin{tabular}{|ll|}
\hline GAPDH-PF & GAAGGTCGGTGTGAACGGAT \\
\hline GAPDH-P R & TTCCCATTCTCGGCCTTGAC \\
\hline IL2-PF & TACAAGAACCCGAAACTGACTCG \\
IL2-PR & ACATGAAGGTAGTCTCACTGCC \\
\hline IL12A-PF & CCTTGCACTTCTGAAGAGATTGA \\
\hline IL12A-PR & ACAGGGCCATCATAAAAGAGGT \\
IL12B-PF & ACCCTGACCATCCAAGTCAAA \\
\hline IL12B-PR & TTGGCCTCGCATCTTAGAAAG \\
\hline
\end{tabular}

\section{Table2 qPCR primer design}

\subsubsection{Proliferation activity of NK92-IL2-IL12 cell line in vitro.}

Collect NK92 cells in logarithmic growth phase (a-MEM culture medium, 12.5\% FBS, 12.5\% horse serum, 1\% penicillin streptomycin, 50U/ml IL2), adjust cell density to $1 \times 10^{\wedge} 5 / \mathrm{ml}$, per well, add $100 \mathrm{ul}\left(10^{\wedge} 4\right)$ to 96 -well plate, culture at $37^{\circ} \mathrm{C}, 5 \% \mathrm{C0} 2$ incubator for 6 hours, 12 hours, 24 hours, 48 hours and then add IOul CCK8, to each well to mix evenly. After being cultured at $37^{\circ} \mathrm{C}$ in $5 \% \mathrm{CO} 2$ incubator for 2 hours, the OD value of $450 \mathrm{~nm}$ wavelength of each well was determined by enzyme labeling instrument. The calculation method of cytotoxic proliferative activity: the average value of three parallel holes was obtained, and the cytotoxic activity of NK was calculated according to the following methods. Cell proliferation activity = [experimental group OD value-blank medium OD value] / [control group OD value-blank medium OD value] $\times 100 \%$.

\subsubsection{Cytotoxicity of NK92-IL2-IL12 cells}

Collect K562 cells and NK92 cells in logarithmic growth phase (a-MEM medium, 12.5\% FBSJI 12.5\% horse serum, 1\% penicillin streptomycin, 50U/ml IL2). K562 cells were used as target cells, and the cell density was adjusted to $2 \times 10^{\wedge} 4 / \mathrm{ml}$;NK cells as effector cells. According to different effector-target ratio, the cell density was adjusted to $2 \times 10^{\wedge} 5 / \mathrm{ml}$, according to gradient dilution to $0.25 \times 10^{\wedge} 5 / \mathrm{ml}, 0.5 \times 10^{\wedge} 5 / \mathrm{ml}, 1 \times 10^{\wedge} 5 / \mathrm{ml}$. The effector cells and target cells were mixed according to different effector-target ratios $(1.25,1.25,1.25,2.5$ and 2.5 , respectively). At the same time, the corresponding target cell hole, effector cell hole and medium blank control hole were set up. There were 3 parallel holes in each group. Add IOul CCK8 (Cell Counting Kit8 to each hole after being cultured in a $5 \% \mathrm{CO} 2$ incubator at $37^{\circ} \mathrm{C}$ for 4 hours), mix well, continue to culture in the incubator at $37{ }^{\circ} \mathrm{C}$ and $5 \% \mathrm{CO} 2 \mathrm{for} 2$ hours, and then determine the OD value of the 450nm wavelength of each well with an enzyme meter. The calculation method of cytotoxic activity: the average value of three parallel holes was calculated, and the cytotoxic activity of NK was calculated according to the following method, which was expressed by killing rate (\%). Cytotoxic activity $=[1$-(effector cell acting pore OD value-effector cell pore OD value) $/$ target cell pore OD value $\times 100 \%$. 
1.2.5 the proliferation and anti-tumor effect of NK92 in in-vivo mouse tumor models.

1. A total of 246 -week-old SCID female mice with an average weight of about 20 Mel $23 \mathrm{~g}$ were selected (provided by Jiangsu Jijiao Pharmaceutical Co., Ltd.). ) they were randomly divided into three groups: PBS group, experimental group NK92 group and control group NK92 cell group, with 8 rats in each group, and were fed in the Animal Experimental Center of Hangzhou normal University with standardized pathogen-free feeding conditions; all the research procedures involved in animals met the animal ethical standards of this research institution. $200 \mu$ I cells containing $2 \times 106 \mathrm{HT}-29$ cells labeled with red fluorescent protein were injected subcutaneously on the right side of each group to form tumor. These labeled HT29 cells expressed red fluorescent protein. As mentioned earlier, the model of SCID mice bearing colon tumor was established and fed routinely. The tumor size was measured twice a week. The tumor volume was calculated according to the formula $\mathrm{V}=0.52 \times \mathrm{L} \times \mathrm{W} 2$ ). "L" represents the longest diameter of the tumor, and "W" is the transverse diameter perpendicular to the longest diameter. After the tumor volume reached about $8 \mathrm{~mm} 3$, the experimental group was injected with $4 \times 106 \mathrm{NK} 92-\mathrm{IL}-2$-IL-12 cells, while the control group was injected with $4 \times 106$ wild NK92 cells, both of which were washed with PBS before injection. The blank control group was only injected with $100 \mu$ I PbS;. NK92 cells were injected into the tumor of mice every 48 hours in 2 weeks. Within 8 hours after each injection, the fluorescence distribution of NK92 cells in the tumor of the two groups was observed by using the in vivo imaging system (IVIS) (Xenogen,Caliper Life Sciences,Hopkinton,MA). The carestream MI software (Shanghai) was used to draw a constant target area on the tumor area, the fluorescence signal intensity was measured, and the experiment of unit total light intensity / cm2 / sr ( $\mathrm{p} / \mathrm{s} / \mathrm{cm} 2 / \mathrm{sr})$, was repeated twice. Four weeks later, three mice in each group were killed, and the tumor tissue of one meter in the center of the tumor was taken. Rabbit anti-human CD 56 antibody and sheep anti-rabbit IgG antibody (cy3 red fluorescence) labeled NK92 cells, and DAPI staining nucleus (blue fluorescence) was detected by immunofluorescence. The number of NK cells was compared in 5 visual fields under $400 x$ magnification. The rest of the tumor-bearing mice died of natural disease, and the survival time was recorded.

\subsubsection{Statistical analyses}

The data was showed as mean \pm SD $(x \pm s)$. One-way and two-way analysis of variance analyses were applied to compare the sample means of test groups and control groups. The results of the CCK8l-assay and results of the in-vivo migration were analyzed using Student's $t$-test. A log-rank test was used for analyses of the survival data. $P<0.05$ was considered statistically significant. All statistical analysis was carried out with Prism software version 7.0 (GraphPad Software Inc., San Diego, USA).

\section{Results}

2.1 lentiviral vector plasmids of IL2 and IL12 were successfully constructed.

Plasmid vector sequencing: plasmid DNA was extracted and sequenced. The sequencing primer is: CAGGAAGAGGGCCTATTTCCC. The partial sequencing results of the skeleton of PUC57, are as follows, and the sequences in the three boxes are the gRNA target sequences of IL2, IL12A and IL12B in turn. The alignment results show that the lentiviral vector plasmids of IL2 and IL12 are constructed successfully.

GGTCTCAACCG GGATCTCCTCAAGTGTCCCCGTTTTAGAGCTAGAAATAGCAAGTTAAAATAAGGCTAGTCCGTTATCAACTTGAAAAAGTGGCACCGAGTCGGTGCTTT

ACC

The green fluorescence expression level of 293T cells was higher after lentivirus transfection, and the transfection rate was more than $99 \%$ (Figure 1c).

Fig1a-b The plasmid profile of LW415 and LW593

Fig.1c. The transfection efficiency of 293T cells using fluorescent inverted microscope.

The left figure is the transfection efficiency of 293 T cell by control vector(PHS-AVR-LW415) using fluorescent inverted microscope.

The right figure is the transfection efficiency of 293 T cell by experimental vector(PHS-AVR-LW593) using fluorescent inverted microscope.

\subsection{Establishment of NK92 cell line stably overexpressing IL2 and IL12.}

1) lentiviral vector plasmid infected NK92 cells: NK92 cells were infected with lentivirus extract and concentrated. 48 hours after infection, the green fluorescence expression level of the cells was shown (Figure 2a), and the infection rate was about $50 \%$. After repeated infection at intervals and repeated screening of puromycin, the positive rate was about $80 \%$.

2) the transcriptional expression level of IL2/IL12 mRNA in NK92 cells detected by IL2/IL12 mRNA fluorescence quantitative PCR was significantly higher than that in wild-type NK92 cells after 48 hours of lentivirus transfection (Figure 2 b), and the fragment size was confirmed to be the target gene by gel electrophoresis (Figure 2c).

2.3The expression of IL2/IL12 protein in NK92 was detected by ElISA.

The expression of IL2/IL12 protein in NK92 cells of modified group was significantly higher than that of wild type NK92 (Fig. 2d).

Fig 2a. Generation of NK92 cells simultaneously overexpressing IL-2 and IL-12. The NK92 cells were infected with concentrated experimental group(obtained from PHS-AVR-LW593 transfect 293-T cells ) virus solution and control group virus solution (obtained from PHS-AVR-LW415 cells)respectively.

Fig 2b.Compared with NK92 infected with the control virus solution,NK92 cells infected by the experimental group virus highly expressed IL2 and IL12 at the mrna level(p[0.001). 
Fig 2c.The size of qPCR products is about 200bp by gel electrophoresis experiment, which is consistent with the size of PCR products designed by primers

Fig.2d The left figure『The NK cells in the experimental group had a higher expression of IL2 than the control NK cells and the complete medium measured by ELISA. The data was measured repeatedly for three times and the average value was obtained. Results are expressed as means of triple experiments per treatment.

The right figure:The NK cells in the experimental group had a higher expression of IL 12 than the control NK cells and the complete medium measured by ELISA. Wild type NK92 control group and complte medium group could not detect the content of IL-12 protein. Results are expressed as means of triple experiments per treatment.

2.4 Killing effect of NK92 cells on tumor target cells.

In the experimental group, the killing activity of NK92 cells to K562 tumor target cells was significantly stronger than that of ordinary NK92 cells. the killing activity of NK cells to K562 cells in group A was higher than that in group B, and the killing activity of NK92 cells against K562 cells increased gradually with the effector-target ratio from 1.25 to $10(P<0.05)$. The result is shown in Figure $3 a$.

2.5 Comparison of proliferative activity of NK92. The result is shown in Figure3b.

Fig3a. Comparison of the killing activity of NK92 cells against K562 cells showed that the killing activity of the IL2/IL12 modified group was significantly higher than that of the wild group, and was proportional to the effective target ratio[*a $[0.05 \square$.Results are expressed as means of triple experiments per treatment.

Fig.3b Comparison of proliferative activity of NK92. Compared with the control group, the test

group has stronger NK cell proliferation activity.lt gradually increased with incubation time, most amplified at 48 hours, about 1.75 times that of the control group. (The test group is the NK cell group overexpressing IL-2-IL-12, and the control is the wild type NK cell group. $\square$

2.6 In the experimental group, NK92 continued to proliferate and its activity increased in tumor-bearing mice.

2.6.1 After 4 weeks, the expression of green fluorescence in tumor was significantly higher than that at 2 weeks (at the end of injection of NK92-IL2-IL12 cells) (Figure 4), indicating that NK92 cells (NK92-IL2-IL12) with overexpression of IL-2 and IL-12 edited by adoptive transfer genes infiltrated and proliferated in the tumor, and SCID mice were injected with HT29 cells labeled with HT29. There is a red fluorescent protein on the right wing. When the tumor grew to about 0.8 cm, NK92-IL-2-IL-12 cells carrying green fluorescent protein were injected into the tumor. The fluorescence imaging system of mice showed that the infiltration of NK cells in the tumor of SCID mice was significantly increased (Fig. 4a). The fluorescence intensity of NK in tumor-bearing mice in the experimental group was also significantly higher than that in the control group (Fig. 4b). According to the oval volume of the tumor (vault $0.52 \times L \times$ W2), the size of the tumor in the NK92-IL-2-IL-12 cell group was significantly smaller than that in the control group (Fig.4c). Combined with the results of figure 6 (increased cytotoxicity of NK92 cells in the experimental group), it could be seen that the killing activity of NK92-IL-2-IL-12 cells in the tumor was significantly stronger than that in the control group, and the survival time of the experimental group was significantly prolonged (Fig.4d). It can be seen that the increase in the number and activity of NK92 cells reduced the tumor load and prolonged the survival time of tumor-bearing mice.

Fig.4a-b:Injection of NK92 cells with overexpression of IL-2 and IL-12 increased intratumoral NK cell infiltration

Fig.4a shows the infiltration region of NK92 cells carrying GFPat different times (LW593 NK92 for test Scid mice,LW415-NK92 for control Scid mice.). Color represents the intensity of the GFP protein carried by NK92 cells, red indicates the strongest.Compared with the control group, the test group had more NK cells infiltrated into the tumor.In addition, the number of NK cells infiltrating in the tumor of the test group was significantly more than four weeks after injection than two weeks, indicating that the experimental group had stronger NK cell proliferation activity in vivo.

Fig.4b Compared with the control NK92 group the fluorescen intensity of tumor area was

significantly increased in the test NK92 group.0p<0.00010

Fig4c-d.Reduced tumor burden and prolonged survival in mice bearing tumors treated with adoptively transferred ex vivo LW590-nk92 cells.(Test group:LW590-nk92 group, Control group:LW415-nk92 group)

Fig.4c shows the tumor size of SCID mice injected with nk92 cells intravenously from the tail, which proves that compared with the pbs group and the control nk92 group, the tumor burden of mice in the test nk92 group is significantly reduced. $(* p<0.05)$.

Fig.4d shows the survival period of tumor-bearing mice in the test group is significantly longer than that in the pbs group and control group.(PD0.01)

2.6.2 the red fluorescence of rabbit anti-human CD56 and sheep anti-rabbit cy3 labeled NK92 cells in lentivirus transfection group was more obvious than that in wild type tumor, and continued to express green fluorescence (Figure 5).

Fig 5.Comparison of fluorescence expression rate of NK92 cells in tumors of mice

\section{3 discussion}

Inhibitory receptor (KIR) [17]and activated receptor (KAR) [18]were expressed on the surface of NK cells. For the autologous cells with normal expression of

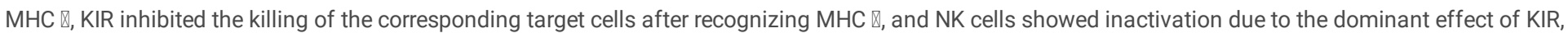


while the expression of MHC class I molecules on the surface of tumor cells decreased or lost, and NK cells showed killing effect due to the dominant effect of KAR. Therefore, NK cells can directly recognize and kill tumor cells without pre-sensitization[19], and the anti-tumor immunity of NK cells is not only efficient, but also has the unique advantages of broad anti-tumor spectrum, individual versatility and immediacy, and can become an important part of general, rapid and efficient cellular immune agents and comprehensive therapy[20, 21].

NK92 cells belong to CD56+ CD16- NK cells, which have cytotoxicity and are not involved in antibody-dependent cell-mediated cytotoxicity ((ADCC)). Because of its safe and effective anti-tumor therapy in vivo, it has been used in the clinical study of adoptive immunotherapy of tumor, and has entered phase $₫-\varangle$ clinical trials, and has been successfully used in clinical treatment of lung cancer, glioma and other clinical treatments (https://clinicaltrials.gov/). Studies have found that the fine number of NK infiltrated in the tumor[22-25] and the improvement of tumor killing activity are very important for the curative effect[26, 27]. In this study, NK92 can proliferate sustainably and enhance its anti-tumor activity by up-regulating the autocrine or paracrine of IL-2, IL-12 and other factors.

Crispr/cas9 gene editing technology has been widely used in the field of gene editing in recent years, and has been successfully applied in the fields of upregulating gene expression in mammalian cells [16,28], gene therapy for genetic diseases and infectious diseases [14] and so on. In this paper, through the construction of transcriptional activation domain dcas9-vp64, the sgRNA genes of IL2 and IL12 were overlapped by T2A to obtain the gRNA sequence of IL2IL12, and the lentiviral expression plasmid of gRNA was constructed. after transfection of NK92, the inactivated dCas9-VP64 was located in the upstream of IL2 and IL12 expression genes of nuclear DNA, and the two cytokines IL2 and IL12, which play key roles in cell proliferation and killing activity, were upregulated in NK92 cells. They act on NK92 cells through cell autocrine or paracrine, enhance the cytotoxicity and activity of NK92 cells, and achieve selfsustainable proliferation and activity enhancement. In this study, the lentiviral expression plasmid of gRNA was obtained by lentivirus packaging infection, and confirmed by sequencing. After infection and integration, the resistance screening of puromycin was observed by fluorescence microscope to obtain cells with a purity of more than $80 \%$. The qPCR and ELISA detection of NK92 cells in each group proved that cytokines IL2 and IL12 were successfully overexpressed in NK92 cells and secreted to extracellular. It is proved that the target cell line of NK92-IL2-IL12 was successfully constructed in this study, which can maintain the sustained and stable overexpression and release of IL2 and IL12. Here, we used CCK-8 method to detect that the proliferation function of this cell increased by 1.75 times. Through the detection of in vivo imaging system and tumor immunohistochemistry in mice for 4 weeks, it was confirmed that the NK92-IL2-IL12 cell line continued to proliferate and enhance its activity in the tumor-bearing mice, so that the general condition of the experimental mice was improved and the survival time was prolonged.

In short, through gene editing technique, NK92 consistently overexpressed IL2 and IL12 cytokines, while IL2 and IL12 cytokines act on peripheral cells and autologous cells through autocrine to promote the proliferation and anti-tumor effect of NK92 cells. In this study, we explored the function of NK92 cells with overexpression of two kinds of cytokines, and found that NK92 cells had stronger anti-tumor activity on the basis of their proliferation ability enhanced greatly. As a result, a new type of NK cells with sustained proliferation and significantly enhanced anti-tumor activity was successfully developed.

\section{Declarations}

\section{Acknowledgements}

Thanks to Hangzhou Normal University Piao Zhenghao teacher provide NK92 cell and NK92 cell culture

technology, thanks to the staff of Hangzhou Normal University affiliated hospital translational medicine platform to provide technical support.

(We sincerely thank Professor Z.H. Piao for his technical help in this work.Also,We acknowledge---)

\section{Funding}

The manuscript(this work) was supported by major science and technology project of zhejiang province 『china

(Project number:2017C03053).

\section{Compliance with ethical standards}

All other authors declare no conflflict of interest.

\section{Ethical approval}

All procedures performed in studies involving animals were in accordance with the ethical standards of

the institution or practice at which the studies were conducted. This article does not contain any studies with human participants performed by any of the authors.

\section{Open Access}

This article is distributed under the terms of the Creative Commons Attribution 4.0 International

License (http://creativecommons.org/licenses/by/4.0/), which permits unrestricted use, distribution, and

reproduction in any medium, provided you give appropriatecredit to the original author(s) and the

source, provide a link to theCreative Commons license, and indicate if changes were made. 


\section{References}

1. 1.Han B,Mao F. Y, Zhao Y. L,Lv Y. P ,et al(2018)Altered NKp30, NKp46, NKG2D, and DNAM-1 Expression on Circulating NK Cells Is Associated with Tumor Progression in Human Gastric Cancer.Journal of immunology research, 2018:6248590. https://doi.org/10.1155/2018/6248590

2. Orange JS, Ballas ZK囚2006】. Natural killer cells in human health and disease. Clin Immunol 118(1):1-10. https://doi.org/10.1016/j.clim.2005.10.011

3. Fehniger TA, Cai SF, Cao X et al(2007) . Acquisition of murine NK cell cytotoxicity requires the translation of a pre-existing pool of granzyme B and perforin mRNAs. Immunity 26(6):798-811. https://doi.org/10.1016/j.immuni.2007.04.010

4. Jamieson AM, Isnard P, Dorfman JR(2004). Turnover and proliferation of NK cells in steady state and lymphopenic conditions. J Immunol 172(2):864-70. https://doi.org/10.4049/jimmunol.172.2.864

5. Watanabe A, Araki K, Harimoto $\mathrm{N}$ et al(2018). D-dimer predicts postoperative recurrence and prognosis in patients with liver metastasis of colorectal cancer. Int J Clin Oncol 23(4):689-97. https://doi.org/10.1007/s10147-018-1271-x

6. Tam YK, Miyagawa B, Ho VC, Klingemann HG(1999). Immunotherapy of malignant melanoma in a SCID mouse model using the highly cytotoxic natural killer cell line NK-92. J Hematother 8(3):281-90. https://doi.org/10.1089/106161299320316

7. Jounaidi Y, Cotten JF, Miller KW(2017). Tethering IL2 to Its Receptor IL2Rbeta Enhances Antitumor Activity and Expansion of Natural Killer NK92 Cells. Cancer Res 77(21):5938-51. https://doi.org/10.1158/0008-5472.CAN-17-1007

8. Wolf AM, Rumpold H, Reimer D. Wolf D(2010). High IL-12 p35 and IL-23 p19 mRNA expression is associated with superior outcome in ovarian cancer. Gynecol Oncol 118(3):244-50. https://doi.org/10.1016/j.ygyno.2010.05.024

9. Alhusseini M, Samantray J(2017) . Hypothyroidism in Cancer Patients on Immune Checkpoint Inhibitors with anti-PD1 Agents: Insights on Underlying Mechanisms. Exp Clin Endocrinol Diabetes 125:267-269.https://doi.org/10.1055/s-0042-119528

10. Ahmed KK, Geary SM, Salem AK(2017). Surface engineering tumor cells with adjuvant-loaded particles for use as cancer vaccines. J Control Release 248:1-9 https://doi.org/10.1016/j.jconrel.2016.12.036

11. Ishino Y, Shinagawa H, Makino K(1987) .Nucleotide sequence of the iap gene, responsible for alkaline phosphatase isozyme conversion in Escherichia coli, and identification of the gene product. J Bacteriol 169(12):5429-5433. https://doi.org/10.1128/jb.169.12.5429-5433.1987.

12. Jinek M, Chylinski K, Fonfara I, Hauer M(2012).A programmable dual-RNA-guided DNA endonuclease in adaptive bacterial immunity. Science 337(6096):816-821.https://doi.org/10.1126/science.1225829

13. Klingemann H, Boissel L, Toneguzzo F(2016). Natural Killer Cells for Immunotherapy - Advantages of the NK-92 Cell Line over Blood NK Cells. Front Immunol 7:91. https://doi.org/10.3389/fimmu.2016.00091

14. Jinek M, East A, Cheng A, Lin S, Ma E, Doudna J(2013). RNA-programmed genome editing in human cells. Elife. 2013;2:e00471.https://doi.org/10.7554/eLife.00471

15. Polstein LR, Gersbach CA(2015). A light-inducible CRISPR-Cas9 system for control of endogenous gene activation. Nat Chem Biol 11(3):198-200. https://doi.org/10.1038/nchembio.1753

16. Konermann S, Brigham MD, Trevino AE et al(2015). Genome-scale transcriptional activation by an engineered CRISPR-Cas9 complex. Nature 517(7536):583-588. https://doi.org/10.1038/nature14136

17. Hsu KC, Keever-Taylor CA, Wilton A et al(2005). Improved outcome in HLA-identical sibling hematopoietic stem-cell transplantation for acute myelogenous leukemia predicted by KIR and HLA genotypes. Blood 105(12):4878-84.https://doi.org/10.1182/blood-2004-12-4825

18. Kim J, Chang CK, Hayden T, Liu FC, Benjamin J, Hamerman JA et al(2007). The activating immunoreceptor NKG2D and its ligands are involved in allograft transplant rejection. J Immunol 179(10):6416-20. https://doi.org/10.4049/jimmunol.179.10.6416

19. Morvan MG, Lanier LL(2016). NK cells and cancer: you can teach innate cells new tricks. Nat Rev Cancer 16(1):7-19. https://doi.org/10.1038/nrc.2015.5

20. Zhang QF, Yin WW, Xia Y, Yi YY, He QF, Wang X et al(2017). Liver-infiltrating CD11b(-)CD27(-) NK subsets account for NK-cell dysfunction in patients with hepatocellular carcinoma and are associated with tumor progression. Cell Mol Immunol 14(10):819-829. https://doi.org/10.1038/cmi.2016.28

21. Cheng M, Chen Y, Xiao W, Sun R, Tian Z(2013). NK cell-based immunotherapy for malignant diseases. Cell Mol Immunol 10(3):230-52. https://doi.org/10.1038/cmi.2013.10

22. Kremer V, Ligtenberg MA, Zendehdel R, Seitz C, Duivenvoorden A, Wennerberg E et al(2017). Genetic engineering of human NK cells to express CXCR2 improves migration to renal cell carcinoma. J Immunother Cancer 5(1):73.https://doi.org/10.1186/s40425-017-0275-9

23. Childs RW, Berg M(2013). Bringing natural killer cells to the clinic: ex vivo manipulation. Hematology Am Soc Hematol Educ Program 2013:234-46. https://doi.org/10.1182/asheducation-2013.1.234

24. Ruggeri L, Capanni M, Urbani E, Perruccio K, Shlomchik WD, Tosti A et al(2002). Effectiveness of donor natural killer cell alloreactivity in mismatched hematopoietic transplants. Science 295(5562):2097-2100. https://doi.org/10.1126/science.1068440

25. Zhang Q, Zhang H, Ding J, Liu H, Li H, Li H et al(2018). Combination Therapy with EpCAM-CAR-NK-92 Cells and Regorafenib against Human Colorectal Cancer Models. J Immunol Res 2018:1-11.https://doi.org/10.1155/2018/4263520

26. Roda JM, Joshi T, Butchar JP, McAlees JW et al(2007). The activation of natural killer cell effector functions by cetuximab-coated, epidermal growth factor receptor positive tumor cells is enhanced by cytokines. Clin Cancer Res 13(21):6419-6428. https://doi.org/10.1158/1078-0432.CCR-07-0865

27. Bryceson YT, Rudd E, Zheng C et al(2007). Defective cytotoxic lymphocyte degranulation in syntaxin-11 deficient familial hemophagocytic lymphohistiocytosis 4 (FHL4) patients. Blood. 2007;110(6):1906-15. https://doi.org/10.1182/blood-2007-02-074468

28. Auer TO, Duroure K, De Cian A(2014). Highly efficient CRISPR/Cas9-mediated knock-in in zebrafish by homology-independent DNA repair. Genome Res 24(1):142-153.https://doi.org/10.1101/gr.161638.113

Page $8 / 13$ 
Figures

a

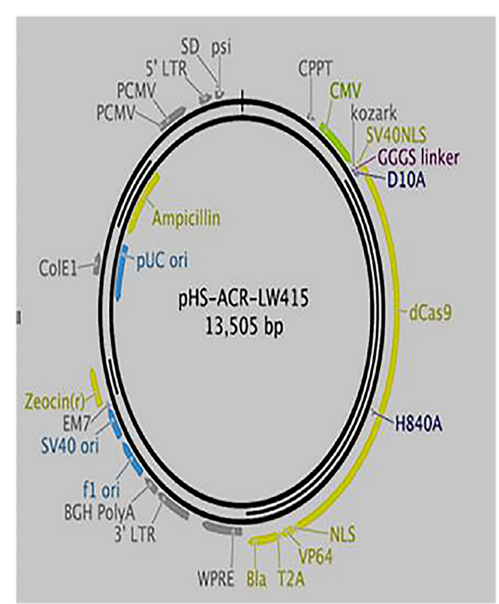

C

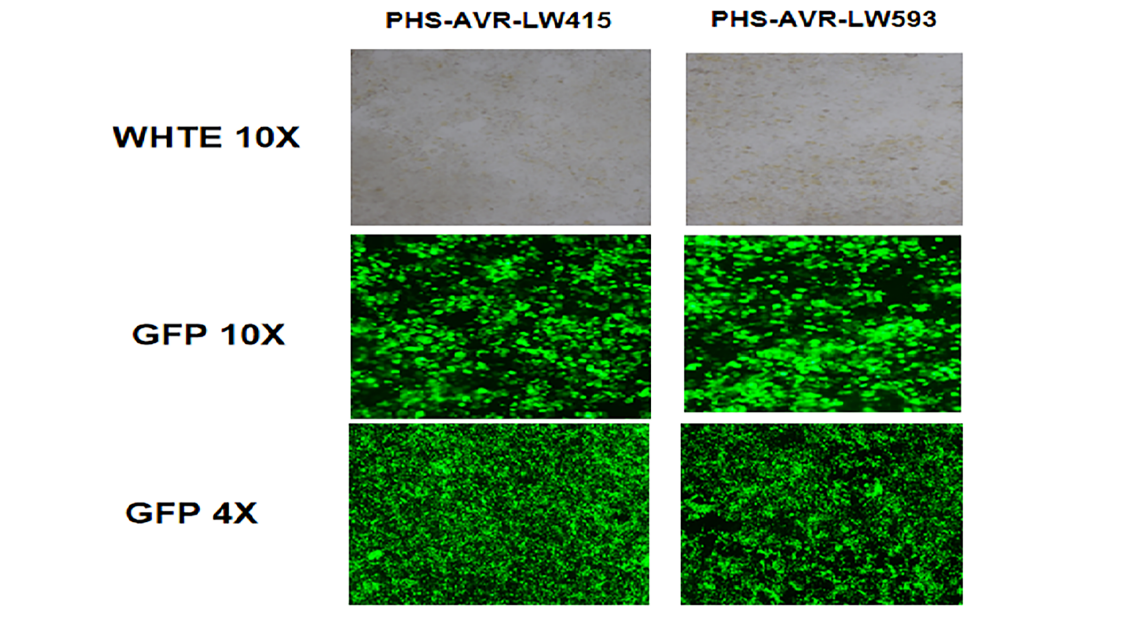

b

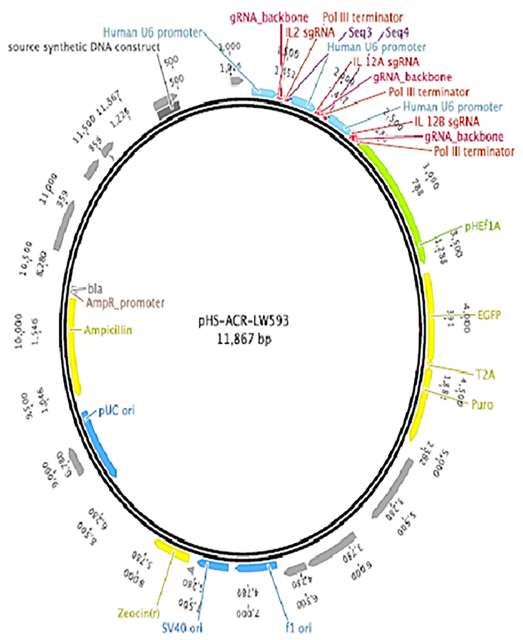

Figure 1

Fig1a-b The plasmid profile of LW415 and LW593 Fig.1c. The transfection efficiency of 293T cells using fluorescent inverted microscope. The left figure is the transfection efficiency of 293T cell by control vector(PHS-AVR-LW415) using fluorescent inverted microscope. The right figure is the transfection efficiency of 293T cell by experimental vector(PHS-AVR-LW593) using fluorescent inverted microscope. 

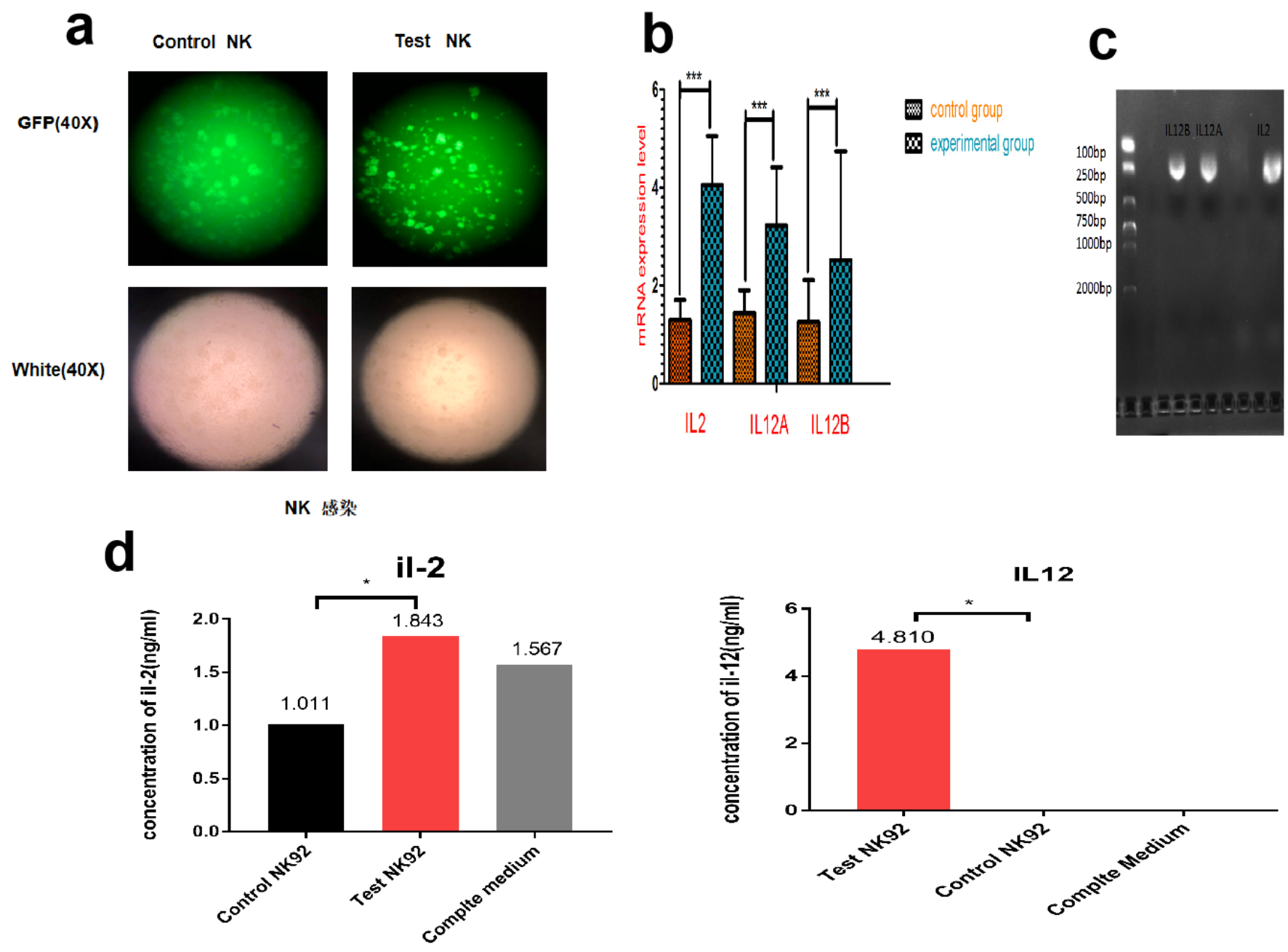

\section{Figure 2}

Fig 2a. Generation of NK92 cells simultaneously overexpressing IL-2 and IL-12. The NK92 cells were infected with concentrated experimental group(obtained from PHS-AVR-LW593 transfect 293-T cells ) virus solution and control group virus solution (obtained from PHS-AVR-LW415 cells)respectively. Fig 2 b. Compared with NK92 infected with the control virus solution,NK92 cells infected by the experimental group virus highly expressed IL2 and IL12 at the mrna level(p囚0.001). Fig 2c. The size of qPCR products is about 200bp by gel electrophoresis experiment, which is consistent with the size of PCR products designed by primers 


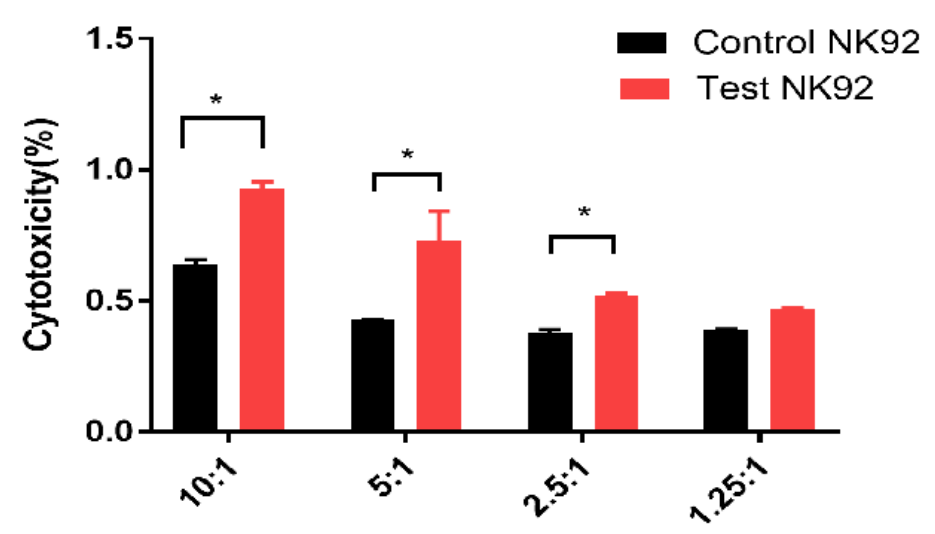

Effector:target ratio

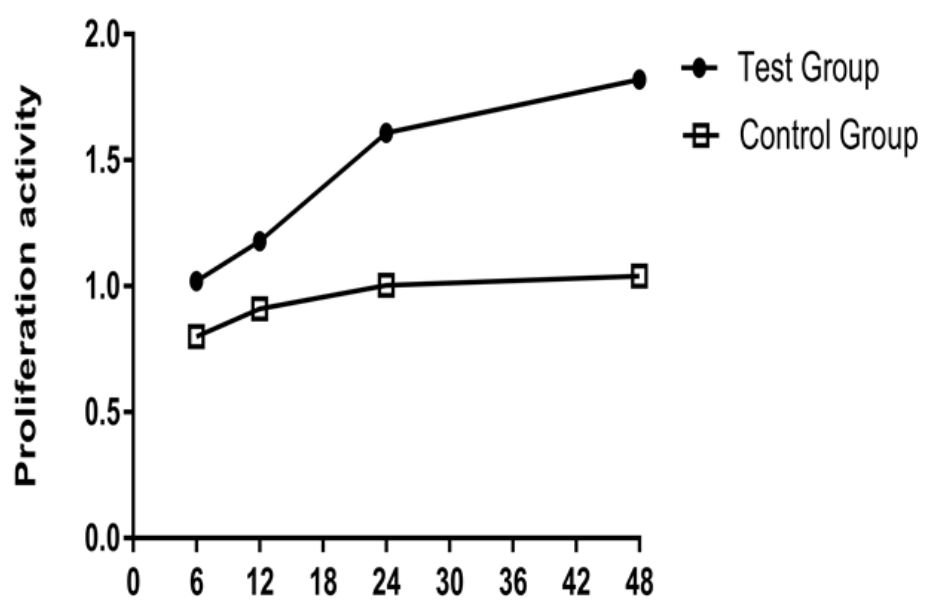

Time(h)

Figure 3

Fig3a. Comparison of the killing activity of NK92 cells against K562 cells showed that the killing activity of the IL2/IL12 modified group was significantly higher than that of the wild group, and was proportional to the effective target ratio ${ }^{*} \mathrm{p} \otimes 0.05 \rrbracket$.Results are expressed as means of triple experiments per treatment. Fig.3b Comparison of proliferative activity of NK92. Compared with the control group, the test group has stronger NK cell proliferation activity.It gradually increased with incubation time, most amplified at 48 hours, about 1.75 times that of the control group. (The test group is the NK cell group overexpressing IL-2-IL-12, and the control is the wild type NK cell group.『 
a

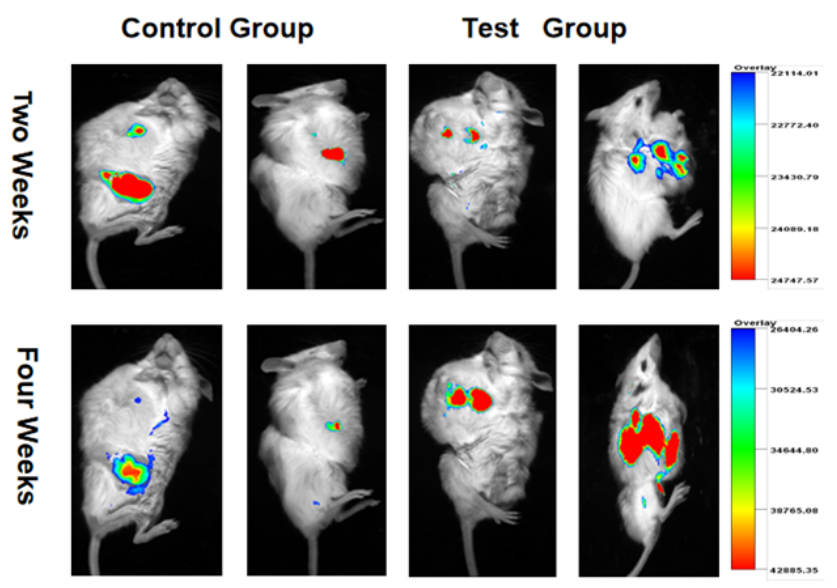

C

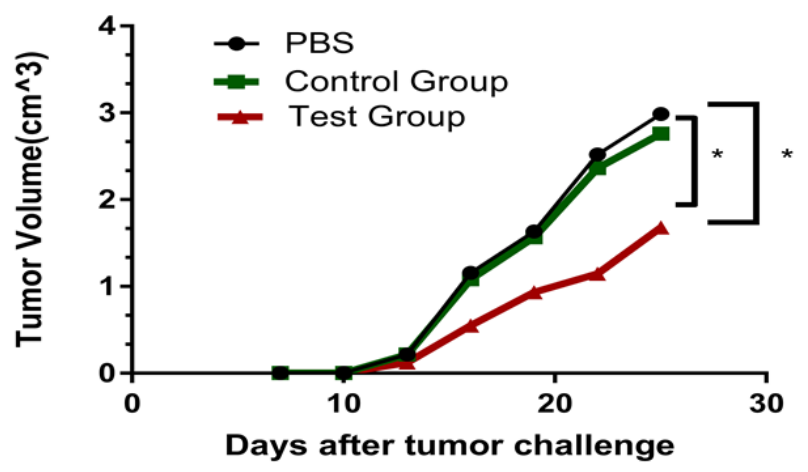

b

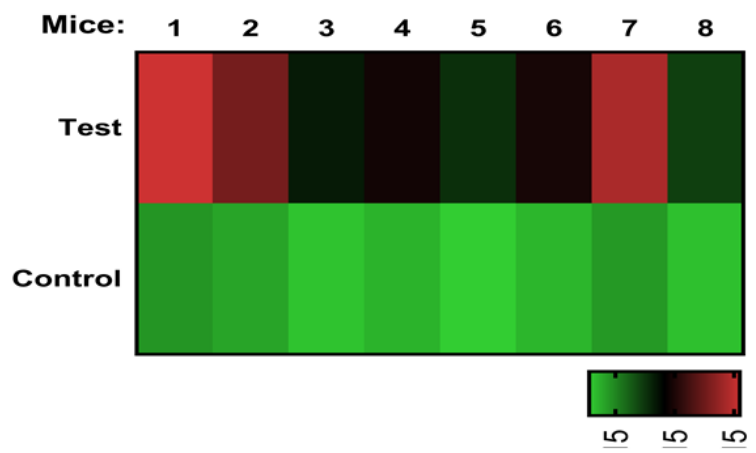

d

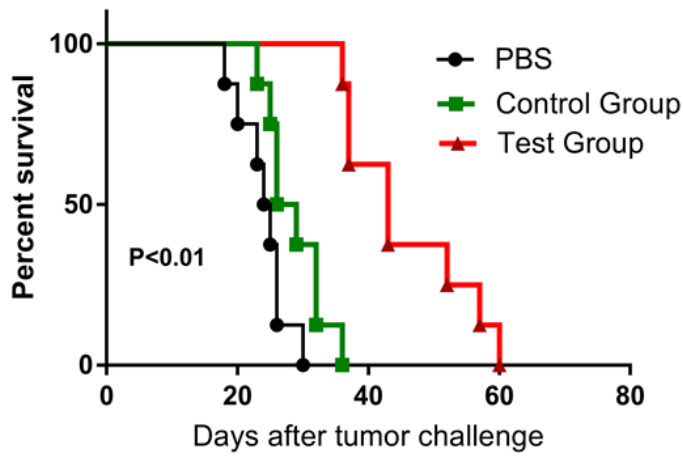

Figure 4

Fig.4a-b:Injection of NK92 cells with overexpression of IL-2 and IL-12 increased intratumoral NK cell infiltration Fig.4a shows the infiltration region of NK92 cells carrying GFP at different times (LW593 NK92 for test Scid mice,LW415-NK92 for control Scid mice.). Color represents the intensity of the GFP protein carried by NK92 cells, red indicates the strongest. Compared with the control group, the test group had more NK cells infiltrated into the tumor. In addition, the number of NK cells infiltrating in the tumor of the test group was significantly more than four weeks after injection than two weeks, indicating that the experimental group had stronger NK cell proliferation activity in vivo. Fig.4b Compared with the control NK92 group the fluorescen intensity of tumor area was significantly increased in the test NK92 group. $\ \mathrm{p}<0.0001 \otimes$ Fig4c-d.Reduced tumor burden and prolonged survival in mice bearing tumors treated with adoptively transferred ex vivo LW590-nk92 cells.(Test group: LW590-nk92 group, Control group:LW415-nk92 group) Fig.4c shows the tumor size of SCID mice injected with nk92 cells intravenously from the tail, which proves that compared with the pbs group and the control nk92 group, the tumor burden of mice in the test nk92 group is significantly reduced. $(* p<0.05)$. Fig.4d shows the survival period of tumor-bearing mice in the test group is significantly longer than that in the pbs group and control group.(P凶0.01) 


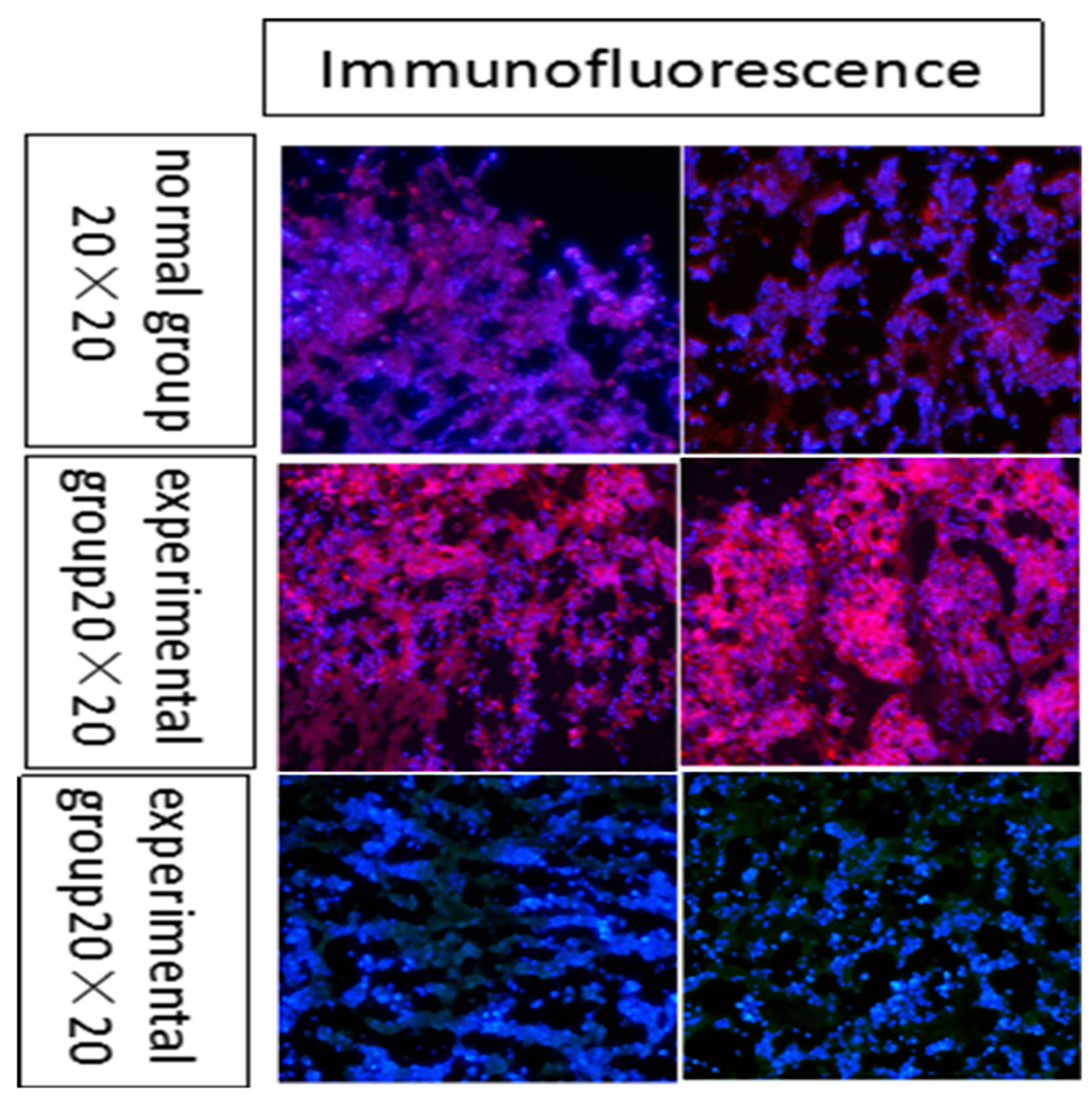

Figure 5

Fig 5. Comparison of fluorescence expression rate of NK92 cells in tumors of mice

\section{Supplementary Files}

This is a list of supplementary files associated with this preprint. Click to download.

- coverletter.doc 